\title{
A simple technique for the generation of institution-specific nomograms for permanent prostate cancer brachytherapy
}

\author{
Kyle J. Lafata, BS', Harry Bushe, MSc², Jesse N. Aronowitz, MD, Prof.2 \\ 'Duke University, Durham, NC, ${ }^{2}$ Department of Radiation Oncology, University of Massachusetts Medical School, Worcester, MA, USA
}

\begin{abstract}
Purpose: Nomograms once had a vital role in prostate brachytherapy practice. Although some of their functions have been assumed by computerized dosimetry, many programs still find them useful to determine the number and strength of seeds to be ordered in advance of the implant. As it has been demonstrated that brachytherapists differ in their implant practices and preferences (in regard to seed distribution and total implanted activity), we propose a simple technique for generating institution-specific nomograms.

Material and methods: Using the data generated by previous implants, we demonstrate a simple technique, utilizing ubiquitous software, for generating nomograms to predict seed number, strength, and total implant activity.

Results: Despite wide variations in the degree of post-implant swelling ( $+12 \%$ to $+56 \%)$, nine of the first ten implants using the generated nomogram have met all target dose parameters.

Conclusions: It is appropriate for each institution to develop nomograms specific to their prostate brachytherapy technique and constraints. Nomograms can estimate the number and strength of seeds to be ordered, and may partially compensate for the effects of implant-induced swelling.

Key words: brachytherapy, medical dosimetry, nomograms, prostate cancer.

\section{Purpose}

Mathematical models (nomograms [1-6], nomographs [7], algorithms [8], reference [9] or look-up tables [10,11], and tie-line charts [12]) have long been used as aids for prostate brachytherapy. They are formulations to 'pre-determine the total required isotope activity for performing transperineal interstitial permanent prostate brachytherapy' from the pre-implant prostate volume [1]. They can be derived from statistical analysis of clinical data $[3-5,10]$, or based upon a theoretical model of the activity needed to subsume a spheroidal volume with a prescription dose cloud $[1,6-8,11,12]$. Although their role has diminished in the era of computerized dosimetry $[13,14]$, nomograms are still used as an independent check of pre-plans, and some brachytherapists utilizing intra-operative planning rely on them to determine the number and strength of seeds in their pre-implant seed order.

As implant preferences (prescription dose, seed distribution pattern, extra-capsular seed placement, organ constraints) differ among institutions, it has been recommended that each institution derive nomograms reflective of their program's implant philosophy $[3,15]$. We relate a simple method, utilizing ubiquitous spreadsheet software, to generate institution-specific nomograms for the calculation of seed number and strength for permanent prostate brachytherapy.

\section{Material and methods}

The process presented here relies on the analysis of previous implants. For purposes of illustration, we present the process we used to generate a nomogram based on the first 40 consecutive Cs-131 ( $\left.{ }^{131} \mathrm{Cs}\right)$ implants performed when our program transitioned to caesium sources (this study, including review of patient records, has been approved by our institution's review board). The implants had been pre-planned, without intentional placement of extracapsular seeds. Post-implant dosimetry was based upon CT scans performed two weeks after implantation.

To generate a nomogram by this process, you will need: 1. Spreadsheet software; we used Excel 2011 for Mac, 14.1 (Microsoft Corporation, Redmond, WA, USA).

2. Post-implant dosimetry for 30 or more prostate implants, that had been analyzed by prostate brachytherapy dosi- 
Table 1. The post-implant dosimetric outcomes

\begin{tabular}{cccc} 
& Range & Median & $\begin{array}{c}\text { Standard } \\
\text { deviation }\end{array}$ \\
\hline $\mathrm{D}_{90}$ & $101-124 \%$ & $112 \%$ & 7.4 \\
\hline $\mathrm{V}_{150}$ & $42-70 \%$ & $52 \%$ & 9.0 \\
\hline $\mathrm{R}_{100}$ & $0.00-0.78 \mathrm{~mL}$ & $0.05 \mathrm{~mL}$ & $0.29 \mathrm{~mL}$
\end{tabular}

metry software; we used VariSeed v. 8.0 (Varian Medical Systems, Inc., Palo Alto, CA, USA). The implants should reflect your institution's implant philosophy (prescription dose, loading pattern, use of extracapsular seeds, organ constraints). Both 'full' (monotherapy) and 'partial' (intended to be combined with external radiation) implants can be used.

3. Defined prescription dose and constraints. Ours were: - prescription dose: 110 Gy for monotherapy, 70 Gy for partial implants;

- constraints: a) $\mathrm{D}_{90}$ range: $100-120 \%$ of prescription dose; b) optimal $\mathrm{D}_{90}$ : midpoint of the $\mathrm{D}_{90}$ range (in our case, $110 \%$ of prescription dose; for monotherapy, $121 \mathrm{~Gy}$ ); c) $\mathrm{V}_{150}$ constraint: $\leq 60 \%$ of prostate; d) $V_{100 \text { rectum }}$ constraint: $<0.75 \mathrm{~mL}$.

\section{The process.}

1. Generate a spreadsheet that contains data from patient records and post-implant dosimetry. Column headings are: a) case identifier; b) pre-implant prostate volume; c) number of seeds implanted; d) activity per seed; e) 'actual' total implant activity (the product of columns ' $c$ ' and 'd'); f) 'optimal' total implant activity (see step 2).

2. Determine 'optimal' total implant activity for each implant using the dosimetry software and post-implant dosimetry, manipulate the individual seed strength for each case (including 'partial' implants) so that each implant achieves an optimal $\mathrm{D}_{90}$ (in our case, $121 \mathrm{~Gy}$ ). Do not add, delete, or move seeds. All seeds must be of the same strength. Once the $\mathrm{D}_{90}$ range is achieved, do not further manipulate the strength of seeds if it would lead to a dose distribution that would exceed constraints $\left(\mathrm{V}_{150}\right.$ or $\left.\mathrm{V}_{100 \text { rectum }}\right)$.

3. Using the spreadsheet software, generate Cartesian (scatter) plots with the 'number of seeds' ( $Y$ axis) as a function of 'prostate volume' ( $\mathrm{X}$ axis). Fit to a linear or power regression. Display the equation and $\mathrm{R}^{2}$.

4. Using the spreadsheet software, generate Cartesian (scatter) plots with 'optimal' total activity ( $\mathrm{Y}$ axis) as a function of 'prostate volume' ( $\mathrm{X}$ axis). Fit to a linear or power regression. Display the equation and $\mathrm{R}^{2}$.

5. You now have equations that can predict the appropriate number of seeds and total implant activity for an implant of a prostate of a given volume. Or, you can generate a 'look-up table'. With the equations generated in steps 3 and 4 , create a look-up table for the range of volumes likely to be implanted (say, 20-60 mL).

The columns being:

a) prostate volume (every integer in the range of volumes); b) number of seeds (solve for $Y$, using the equation generated in step 2, where $X$ is the prostate volume);

c) 'optimal' total implant activity (solve for $Y$, using the equation generated in step 3 , where $X$ is the prostate volume);

d) activity per seed (divide column c by column b).

It is advisable to order a few extra seeds to compensate for seed loss or the discovery of a larger than anticipated prostate volume at time of implantation. The nomogram can assist in making appropriate adjustments in total implant activity in the operating theatre if the prostate volume is determined to be different than measured preoperatively (e.g., due to the action of hormonal therapy).

\section{Results}

Our first $10{ }^{131} \mathrm{Cs}$ implants guided by the nomogram generated by this method were analyzed. The post-implant dosimetric outcomes are summarized in Table 1.

Not surprisingly, the degree of post-implant swelling, expressed as the ratio of post-implant prostate volume (on CT performed 2 weeks after implantation) to pre-implant prostate volume (measured by ultrasound) greatly influenced post-implant dosimetry metrics. The median ratio was 1.28 , but the range was 1.12-1.56. The implant with the greatest degree of swelling (ratio 1.56) had the 'coolest' $D_{90}(101 \%)$ and $V_{150}(42 \%)$. The implant with the least swelling (ratio 1.12) had the 'warmest' $\mathrm{D}_{90}(124 \%)$ and $\mathrm{V}_{150}$ $(70 \%)$; the latter was the only implant in which constraints were not met.

The volume of tissue encompassed by the prescription dose on the post-implant CT scans ranged from 1.46 to 1.69 times the pre-implant prostate volume (mean: 1.55, standard deviation: 0.08).

\section{Discussion}

When the clinicians and physicists at New York's Mt. Sinai Hospital began their prostate implant program (1990), they adopted the Memorial Hospital nomogram to determine the total activity to implant for a given prostate volume. It became apparent, however, that the nomogram developed for Memorial's homogenous seed distribution was inappropriate for Sinai's peripherally-loaded implants. In a series of 5 iterations over 5 years, the Sinai program developed an institution-specific nomogram to satisfy their needs [16]. When our institution switched from ${ }^{125} \mathrm{I}$ to ${ }^{131} \mathrm{Cs}$ seeds, we relied on guidelines that had been developed elsewhere. We, too, were disappointed with our dosimetric outcomes and endeavored to develop a nomogram that reflected our implant pattern and dosimetric constraints.

Nomograms had once been used to determine total implant activity, seed number, and spacing [7]. We believe that computer planning has reduced, but not eliminated the utility of nomograms. Nomograms can be used for quality control and to maintain consistency among implant plans. The VariSeed software utilizes a 'Nomogram Planning Module' to generate a complete treatment plan (including total activity, needle, and source positions), but relies on a nomogram entered by the operator. It may be 
unsatisfactory to adopt a 'generic' nomogram obtained from medical literature or provided by a seed distributor, as it has been demonstrated that experienced brachytherapists espousing the same implant philosophy and prescribing to the same dose will implant the same volume with a different number of seeds and total activity [17]. Indeed, the variation in total implant activity can exceed $40 \%$ [15]. It has therefore been recommended that a nomogram be generated to reflect an institution's own practices $[3,15]$.

It has long been appreciated that the edema engendered by prostate implantation can degrade implant quality [18-20]. If the magnitude of swelling could be predicted, it would be a simple matter to compensate by a reciprocal increase in implant strength; but the magnitude is highly variable and unpredictable [18-20]. Two strategies have been commonly used to reduce the impact of swelling on implant quality:

1. Devising a treatment plan in which the target volume encompasses the prostate with a several millimeter margin [21]. Use of our nomogram has resulted in the delivery of prescription dose to a volume $47-69 \%$ greater than the pre-implant prostate volume (coinciding with previously observed day-1 swelling), without exceeding normal tissue constraints or placement of extracapsular seeds.

2. Utilizing intra-operative treatment planning with computer optimization [13]. Unless the brachytherapist orders seeds in bulk (impractical for small programs and those utilizing short half-life radionuclides), a method is required for predicting the number and strength seeds that will be used.

It has been proposed that nomograms can be used to at least partially compensate for implant-induced edema [22]; our experience suggests this to be so. The technique we have proposed for nomogram generation utilizes ubiquitous software; it has been utilized by one of the authors (JNA) for over a decade. His nomogram has been revised whenever his technique was modified or a new radionuclide was adopted (the process facilitates changing of radionuclides, as the post-implant CT scans performed after implantation with the 'old' radionuclide can be used to generate a nomogram for the 'new' one).

Nomograms can be based on either linear or power equations. Historically, power equations have been most often used, but it had previously been demonstrated that, for the range of volumes typically implanted, a power curve is almost 'linear' [23]; our current experience confirms this (Fig. 1). Indeed, the calculated difference between our linear and power equations was less than one seed for prostate volumes between 25 and $45 \mathrm{~mL}$ (data not shown). We suggest that practitioners generate both power and linear equations, and use the equation that has the better fit (higher $\mathrm{R}^{2}$ ). We wish to stress that nomograms may have a supplementary role in treatment planning, and do not diminish the centrality of computer dosimetry in brachytherapy planning.

\section{Conclusions}

Computer dosimetry has diminished, but not eliminated the role of nomograms in prostate brachytherapy. They

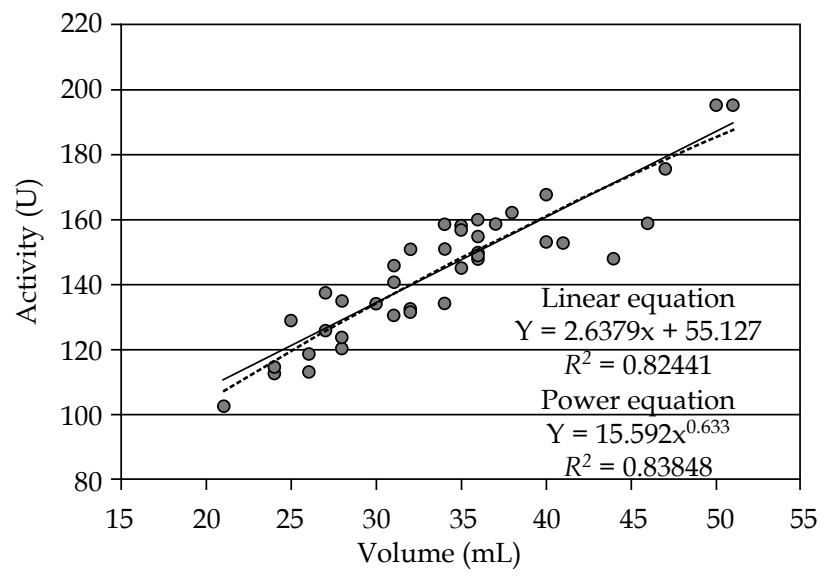

Fig. 1. Excel-generated graph of the relationship between pre-implant prostate volume and optima total implant activity. Both linear (solid line) and power (dashed line) fit formalisms are represented. Although there is a marginally better fit (reflected by a higher $R^{2}$ ) for the power equation, note that curves overlap for the range of volumes most commonly implanted

remain useful for estimating the total activity and number of sources to be implanted. Our experience suggests that they can partially compensate for the unpredictable degree of post-implant edema. Brachytherapists should use nomograms that reflect their own implant practices, tissue constraints, and philosophy. We have demonstrated a simple method for the generation of institution-specific nomograms for permanent prostate brachytherapy.

\section{Disclosure}

Authors report no conflict of interest.

\section{References}

1. Wang X-H, Potters L. A theoretical derivation of the nomograms for permanent prostate brachytherapy. Med Phys 2001; 28: 683-687.

2. D'Souza WD, Lee HK, Palmer MB et al. Is intraoperative nomogram-based overplanning of prostate implants necessary? Int J Radiat Oncol Biol Phys 2003; 56: 462-467.

3. Al-Qaisieh B, Brearley E, Clair SS et al. A study of a pretreatment method to predict the number of I-125 seeds required for prostate brachytherapy. Int J Radiat Oncol Biol Phys 2006; 65: 304-307.

4. Kudchadker RJ, Swanson DA, Kuban DA et al. Is a loose-seed nomogram still valid for prostate brachytherapy in a strandedseed era? Int I Radiat Oncol Biol Phys 2008; 72: 623-627.

5. Langley SEM, Laing RW. 4D brachytherapy, a novel realtime prostate brachytherapy technique using stranded and loose seeds. BJU Internat 2012; 109: s1-6.

6. Li T, Fountain BL, Duffy EW. Physical derivation of nomograms in permanent prostate brachytherapy. Brachytherapy 2010; 9: 50-54.

7. Anderson LL. Spacing nomograph for interstitial implants of I-125 seeds. Med Phys 1976; 3: 48-51.

8. Yu Y, Schell MC. A genetic algorithm for the optimization of prostate implants. Med Phys 1996; 23: 2085-2091.

9. McNeely LK, Stone NN, Presser J et al. Influence of prostate volume on dosimetry results in real-time I-125 seed implantation. Int J Radiat Oncol Biol Phys 2004; 58: 292-299. 
10. Stone NN, Stock RG. Brachytherapy for prostate cancer: Realtime three-dimensional interactive seed implantation. Tech Urol 1995; 1: 72-80.

11. Wu A, Lee C-C, Johnson M, Brown D et al. A new power law for determination of total I-125 seed activity for ultrasound-guided prostate implants: clinical evaluations. Int J Radiat Oncol Biol Phys 2000; 47: 1397-1403.

12. Anderson LL, Moni JV, Harrison LB. A nomograph for permanent implants of palladium-103 seeds. Int J Radiat Oncol Biol Phys 1993; 27: 129-135.

13. Zelefsky MJ, Yamada Y, Cohen G et al. Postimplantation dosimetric analysis of permanent transperineal prostate implantation: Improved dose distributions with an intraoperative computer-optimized conformal planning technique. Int J Radiat Oncol Biol Phys 2000; 48: 601-608.

14. Raben A, Chen H, Grebler A et al. Prostate seed implantation using 3D-computer assisted intraoperative planning vs. a standard look-up nomogram: Improved target conformality with reduction in urethral and rectal wall dose. Int J Radiat Oncol Biol Phys 2004; 60: 1631-1638.

15. Aronowitz JN, Crook JM, Michalski JM et al. Inter-institutional variation of implant activity for permanent prostate brachytherapy. Brachytherapy 2008; 7: 297-300.

16. Stone NN, Stock RG, DeWyngaert JK et al. Prostate brachytherapy: Improvements in prostate volume measurements and dose distribution using interactive ultrasound guided implantation and three-dimensional dosimetry. Radiat Oncol Invest 1995; 3: 185-195.

17. Merrick GS, Butler WM, Wallner KE et al. Vaariability of prostate brachytherapy preimplant dosimetry: a multi-institutional analysis. Brachytherapy 2005; 4: 241-251.

18. Prestidge BR, Bice WS, Kiefer EJ et al. Timing of computed tomography-based postimplant assessment following permanent transperineal prostate brachytherapy. Int J Radiat Oncol Biol Phys 1998; 40: 1111-1115.

19. Waterman FM, Yue N, Corn B et al. Edema associated with I-125 or Pd-103 prostate brachytherapy and its impact on post-implant dosimetry: an analysis based on serial CT acquisition. Int J Radiat Oncol Biol Phys 1998; 41: 1069-1077.

20. Dogan N, Mohideen N, Glasgow GP et al. Effect of prostatic edema on CT-based postimplant dosimetry. Int J Radiat Oncol Biol Phys 2002; 53: 483-489.

21. Waterman FM, Dicker AP. Impact of post-implant edema on V100 and D90 in prostate brachytherapy: Can implant quality be predicted on day 0? Int J Radiat Oncol Biol Phys 2002; 53: 610-621.

22. Potters L, Wang XH, Yamada Y. A nomogram to compensate for intraoperative prostate edema during transperineal brachytherapy. Tech Urol 2000; 6: 99-103.

23. Aronowitz JN, Michalski JM, Merrick GS et al. Optimal equations for describing the relationship between prostate volume, number of sources, and total activity in prostate brachytherapy. Am J Clin Oncol 2010; 33: 164-167. 